\section{Narrativas de usuários de saúde mental em uma experiência de gestão autônoma de medicação}

\author{
Narratives of users of mental health services in \\ an experiment with autonomous management \\ of medication
}

\author{
Narraciones de usuarios de servicios de salud \\ mental dentro una experiencia con la \\ gestión autónoma de medicación
}

Laura Lamas Martins Gonçalves 1 Rosana Teresa Onocko Campos 2

doi: 10.1590/0102-311X00166216

\section{Resumo}

Numerosos estudos apontam a tendência à cronicidade dos tratamentos quando centrados no uso de medicamentos. Esta pesquisa foi realizada em uma grande cidade brasileira, com usuários de Centros de Atenção Psicossocial (CAPS), com o objetivo de avaliar os efeitos da experimentação do Guia pessoal da Gestão Autônoma da Medicação (Guia GAM) na relação desses usuários com seus tratamentos e nas suas ações de participação. Esse instrumento, criado no Canadá, traduzido e adaptado para o Brasil, foi experimentado, por meio de grupos de intervenção, com usuários de CAPS com transtorno mental grave e com história de participação política no campo da saúde. Foram realizados grupos focais e entrevistas em profundidade. As transcrições foram transformadas em narrativas, e foram definidas quatro categorias de análise: sujeitos de experiências radicais de sofrimento; experiências com a medicação; direitos de usuários; participação e militância politica. Com a experimentação do Guia GAM, os usuários expressaram uma tensão entre a reprodução da identidade centrada na doença e a legitimidade da singularidade das suas experiências pessoais. Demonstraram maior conhecimento sobre os medicamentos que tomavam, passaram a reconhecer sua própria expertise, como o uso de medicamentos, e alguns buscaram ajustes no tratamento. Referiram necessidade de serem apoiados nos movimentos de reivindicação dos seus direitos e fortaleceram suas participações enquanto militantes da saúde mental. Conclui-se que a versão brasileira do Guia GAM tem potencial de contribuir com o empoderamento dos usuários, permitindo assim uma interlocução das avaliações de resultados em saúde mental com o cenário internacional.

Serviços de Saúde Mental; Uso de Medicamentos; Participação Cidadã; Autonomia Pessoal

\section{Correspondência}

L. L. M. Gonçalves

Universidade Federal do Maranhão.

Rua Barão de Itapari 155, São Luís, MA 65020-070, Brasil.

laulmg@gmail.com

1 Universidade Federal do Maranhão, São Luís, Brasil.

2 Universidade Estadual de Campinas, Campinas, Brasil. 


\section{Introdução}

No Brasil, o processo de Reforma Psiquiátrica alcançou avanços significativos, com investimentos em recursos humanos e políticas de atenção em saúde mental. Entretanto, em que pese os dispositivos de saúde mental substitutivos ao manicômio, como os Centros de Atenção Psicossocial (CAPS), buscarem implantar o novo modelo assistencial, ainda reproduzem práticas hegemônicas do paradigma que tentam superar 1, funcionando com resquícios da lógica manicomial, em que há predomínio de uma relação marcada pela dominação do profissional da saúde sobre o paciente 2 , com a consequente infantilização do segundo diante de seu processo de tratamento ${ }^{1}$. Nos serviços de saúde mental, predominantemente, são os técnicos que possuem os meios de regulação e funcionamento dos serviços, produzindo desarticulação e enfraquecimento dos usuários, com pouca ou nenhuma possibilidade de gestão das atividades oferecidas, não atuando como sujeitos ativos nos processos decisórios, mas como espectadores passivos do processo de gestão dos serviços e de suas próprias vidas 3,4.

Muitos serviços de saúde mental ainda funcionam reproduzindo uma atenção centralizadora e especializada, cronificada e produtora de cronificação 5,6,7, com um modelo centrado na doença, na sintomatologia e no tratamento medicamentoso 8 , enfraquecendo a construção de espaços e dispositivos de cuidado horizontalizados e promotores de maiores graus de autonomia 9 . Os serviços de saúde mental ainda privilegiam o tratamento medicamentoso, reduzindo, muitas vezes, o tratamento aos psicotrópicos, incluindo ainda situações de supermedicação. Pesquisas apontam a falta de informação dos usuários sobre os remédios receitados e a não discussão sobre os efeitos indesejáveis deles, revelando uma baixa apropriação dos usuários dos serviços em relação ao seu tratamento. Há principalmente um baixo conhecimento em relação aos efeitos benéficos e colaterais de qualquer tratamento medicamentoso, reduzindo a capacidade crítica do processo decisório na escolha do tratamento, fazendo com que o poder dessa decisão recaia apenas nos profissionais de saúde 10 .

Percebe-se um crescente processo de medicamentalização 11,12,13,14, problemas que antes eram tratados em sua complexidade e singularidade são classificados como doenças, déficits ou transtornos e medicados. Como consequência, vê-se um aumento significativo do consumo de psicofármacos no Brasil e no mundo 15,16,17,18,19: seu uso e prescrição têm aumentado, não apenas em quantidade, mas também em duração do uso, por períodos, às vezes, maiores do que os preconizados na literatura especializada 20. A participação dos usuários restringe-se, muitas vezes, à mera informação de seus sintomas, ficando, de lado, os múltiplos não ditos do sofrimento 21,22,23.

$\mathrm{O}$ uso de medicamentos psicotrópicos tem sentidos singulares para as diferentes pessoas, e seus testemunhos mostram que são plurais os aspectos simbólicos da medicação e, frequentemente, contraditórios 10. Entretanto, a experiência de adoecimento 24,25,26 e a pluralidade de experiências e significações do uso de medicamentos psicotrópicos para as pessoas continuam sendo raramente levadas em conta nas práticas de cuidados em saúde mental 22,27,28, mesmo sabendo-se que, quando a prescrição medicamentosa é acompanhada de uma disponibilidade maior de diálogo e é negociada com a pessoa, são obtidos os mesmos efeitos com doses menores do que quando predomina um controle externo das prescrições 29.

Além disso, a comunicação entre os profissionais de saúde e usuários sobre o tratamento é deficiente 30 , e alguns estudos 31,32,33 constatam uma assimetria presente nas relações entre trabalhadores e usuários, indicando processos que ainda constrangem o protagonismo e favorecem a manutenção da histórica passividade dos usuários de saúde mental. Mostram que disputa de interesses, hierarquização nas relações de poder, enrijecimento nos modos de conduzir processos participativos podem reduzir ou inviabilizar as possibilidades de protagonismo em saúde mental.

Uma das diretrizes políticas do Sistema Único de Saúde brasileiro (SUS) diz respeito à participação cidadã na gestão do sistema e dos serviços de saúde por meio da inclusão de novos sujeitos nos processos de decisão na saúde. A participação tem sido considerada fator constitutivo das políticas sociais e fundamental para atingir o objetivo de autopromoção e empoderamento das pessoas 34, entendendo-a como um exercício de compartilhamento de poder 35. Entretanto, a efetiva participação dos usuários na produção da saúde é um dos vetores que precisa ser considerado nesse contexto da atenção em saúde mental, já que pouco ainda se avançou na efetiva participação dos usuários, sobretudo nas decisões sobre seus tratamentos. 
No cenário internacional, a participação dos usuários nas decisões relacionadas aos seus tratamentos vem sendo experimentada e discutida há alguns anos. No Reino Unido, os guidelines oficiais do sistema nacional de saúde explicitam o compartilhamento da decisão com os usuários como condição imperativa para o seguimento dos tratamentos de saúde, e a tomada de decisão compartilhada é cada vez mais defendida como um modelo ideal de tomada de decisão de tratamento no encontro médico 36. A decisão compartilhada pressupõe que usuário e prescritor devem compartilhar suas respectivas informações e determinar, de forma colaborativa, o tratamento ideal 37,38,39.

Ao mesmo tempo, a Organização Mundial da Saúde 40,41 tem recomendado a criação e a difusão de grupos de defesa dos usuários, com intuito de fortalecer o empoderamento e o protagonismo desses coletivos na construção das políticas de saúde mental no planejamento, desenvolvimento, monitoramento e avaliação dos serviços. Os movimentos reformistas no campo da saúde mental vêm buscando fortalecer o empoderamento dos usuários com transtornos mentais e o incremento de seu poder de contratualidade na sociedade por meio de propostas de reabilitação psicossocial que passam pelo exercício da autonomia e da cidadania 42.

Mas, em que medida tem sido possível um incremento no poder de contratualidade quando o que se evidencia no contexto dos serviços de saúde mental é a dificuldade em retirar a doença do foco das atenções e entender que o sujeito que sofre é protagonista da sua história, tanto na experiência de adoecer quanto de cuidado de si?

No Canadá, a Gestão Autônoma da Medicação (GAM), uma abordagem que vem sendo desenvolvida desde a década de 1990, reconhece justamente o contexto de utilização pouco crítica dos medicamentos nos tratamentos em saúde mental, bem como o valor simbólico da medicação para aqueles que a utilizam. Defende e apoia pessoas fragilizadas pela experiência dos problemas de saúde mental e, às vezes, também pelo tipo de tratamento oferecido nos serviços de saúde mental. Objetiva a criação de espaços de expressão e de decisão acerca do tratamento e da qualidade de vida, favorecendo uma relação ativa e não mais passiva com o tratamento medicamentoso 22,28,29. A GAM considera os usuários como protagonistas e corresponsáveis do processo de gestão dos medicamentos (da decisão de usar e do modo como usar). Os saberes dos usuários, seus desejos, vontades e opiniões ganham relevância e igualdade de importância com o saber médico. Trata-se de uma aposta em uma prática de atenção em saúde mental que reconhece que o tratamento só pode ser conduzido com a inclusão do usuário no processo decisório das terapêuticas a serem realizadas 22.

Uma de suas ferramentas é o Guia GAM 29, um instrumento que reconhece o direito ao consentimento livre e esclarecido para a utilização da medicação e a necessidade de compartilhamento de decisões entre profissionais e usuários e tem como um de seus objetivos disponibilizar, para os usuários, informações sobre as medicações (efeitos colaterais, doses terapêuticas etc.). Com seu conteúdo dividido em passos, para facilitar a leitura e a reflexão individual ou em grupo, disponibiliza informações sobre medicamentos psicotrópicos (efeitos colaterais, doses terapêuticas) e sobre direitos dos usuários, convidando o leitor a fazer um balanço da própria vida para determinar os aspectos suscetíveis de serem melhorados. Durante esse processo, a medicação pode ou não surgir como um desses aspectos.

A pesquisa de que trata este artigo experimentou o Guia GAM, já traduzido e em processo de adaptação ao Brasil, no qual a negociação e o incremento da participação do usuário na gestão do seu tratamento foram enfatizados pelo entendimento de que a decisão quanto ao melhor tratamento se faz em uma composição entre os saberes do usuário e da equipe de referência, em um exercício de cogestão, de gestão compartilhada do cuidado 22,43,44,45.

\section{Objetivo}

Trata-se de uma pesquisa realizada em uma grande cidade brasileira, com usuários de CAPS, com o objetivo de avaliar os efeitos da experimentação do Guia GAM na relação desses usuários com seus tratamentos e nas suas ações de participação política. 


\section{Metodologia}

O Guia GAM foi trabalhado, por meio da constituição de um grupo de intervenção, com sete usuários de CAPS, com transtorno mental grave e com história de participação política no campo da saúde mental. Foi composto por meio de uma articulação dos pesquisadores com membros de uma associação de usuários, familiares e amigos de usuários da rede de saúde mental da cidade. Foram critérios de inclusão na pesquisa: ser portador de transtorno mental grave, fazer uso de psicofármacos há mais de um ano, ter vontade de participar do grupo, ter boa circulação pela cidade e pela rede de serviços e ter trajetória de participação política no campo da saúde mental. Os critérios de não inclusão foram: recusa em participar ou limitação cognitiva grave.

Os sete usuários participantes eram todos homens, moradores do município em que ocorreu a pesquisa e tinham entre 31 e 50 anos de idade. Desses, inicialmente, quatro e, ao final da pesquisa, cinco eram integrantes de uma Associação de Familiares, Amigos e Usuários de Saúde Mental do município. Outros dois eram representantes dos usuários nos conselhos locais dos serviços que frequentavam.

O grupo de intervenção foi realizado em encontros quinzenais, num total de 18 encontros, e conduzido por pesquisadores. Tinha como objetivo o compartilhamento das experiências com tratamento medicamentoso, a partir dos temas propostos no Guia. Cada participante recebeu um exemplar do Guia. Diversas dinâmicas para leitura e discussão foram utilizadas. Ao final de cada encontro, os pesquisadores registravam as discussões, consideradas também como material empírico da pesquisa. O manejo com o grupo foi tema constante de conversas e trocas entre os pesquisadores, incluindo um espaço regular de supervisão com a coordenação da pesquisa.

Foram realizados também grupos focais 46,47 com participação dos sete usuários, antes (GF0) e depois do grupo intervenção (GF1, grupo focal narrativo), buscando aproximar a experiência dos participantes, com foco nos seguintes temas: uso de medicamentos psiquiátricos na relação com a autonomia e os direitos dos usuários; valorização do contexto do usuário; capacidade de gestão e compartilhamento de decisões (usuário e equipe); direitos do usuário, em especial no que se refere à medicação (acesso, informação, recusa); tomada da palavra (voz do usuário no serviço e na relação médico/paciente); experiência de uso de medicação. No GF1, buscou-se identificar os efeitos do Guia nas narrativas sobre essas experiências.

Ao final de cada grupo focal, a partir das transcrições e da extração de seus núcleos argumentais 48, foi criada uma narrativa pelos pesquisadores que haviam sido os condutores de cada grupo e validada, posteriormente, por outro pesquisador, totalizando três pesquisadores envolvidos nesse processo de criação e validação. As falas dos usuários nos grupos focais foram tomadas como falas de um grupo e destacadas em seus aspectos de composição e concordância, mas também naquilo que, no grupo, compareceu como dúvida, dissenso ou clara discordância a partir da construção de narrativas 48 .

Após o GF1, foi realizado um terceiro grupo focal, o grupo focal narrativo 49,50. No grupo focal narrativo, as narrativas de cada um dos grupos focais (GF0 e GF1) foram apresentadas aos usuários para uma validação final. $O$ grupo focal narrativo permitiu que as mudanças percebidas como efeitos do grupo intervenção fossem discutidas, promovendo simultaneamente a validação e a participação na análise de dados.

Após o grupo focal narrativo, foram realizadas entrevistas individuais, em profundidade 51, com os sete integrantes do grupo interveção como forma de ampliar a investigação sobre o efeito da experimentação do Guia GAM no grupo intervenção, buscando uma aproximação da experiência dos participantes no processo de adoecimento, com o uso de medicamentos psiquiátricos, com a participação em seus tratamentos e com a militância política. As entrevistas foram realizadas por um único pesquisador, totalizando 12 horas de gravação de áudio e 142 páginas de transcrição.

Para a análise e interpretação dos dados, foi utilizado referencial teórico proposto por Ricoeur 52,53,54 e Onocko Campos 48, concebendo as narrativas como um processo de mediação entre o vivido e a possibilidade de inscrevê-lo no social, inserindo a experiência subjetiva em um campo político. As narrativas dão visibilidade à experiência dos sujeitos pesquisados e também trazem a dimensão da experiência do que se vive no desenrolar da pesquisa. A análise final foi obtida a partir da triangulação das entrevistas com as narrativas construídas a partir dos grupo focal. As pesquisadoras, de forma independente e sequencial, fizeram reiteradas leituras flutuantes e cruzamentos, finalizando com uma 
reunião de consenso e validação entre elas 50,55. A postura ético-política da pesquisa foi a de não transformar as vozes em objetos 55 , de modo que a análise não se fez sobre as experiências, mas com elas 50 .

A pesquisa foi aprovada em Comitê de Ética da Faculdade de Ciências Médicas, Universidade Estadual de Campinas sob o parecer CEP/FCM/UNICAMP no 222/2009. Todos os participantes assinaram o Termo de Consentimento Livre e Esclarecido, tendo sido garantidos aos participantes o anonimato e a confidencialidade das informações.

\section{Resultados e discussão}

No processo de análise foram identificadas quatro categorias, apresentadas a seguir.

\section{Sujeitos de experiências radicais de sofrimento}

Nas narrativas dos usuários, comparece uma tensão entre a construção e a reprodução de uma identidade doente versus a legitimidade da singularidade das experiências dos usuários. De modo geral, apontaram o diagnóstico da doença como o reconhecimento das suas experiências radicais de sofrimento, algo que os ajudou a ressignificar positivamente o sofrimento, pois familiares e a sociedade, em geral, passaram a reconhecê-los como sujeitos doentes. Para eles, o ato de ser diagnosticado e medicado foi tomado como uma resposta ao reconhecimento da singularidade da experiência de sofrimento. "Pior é alguém que não acredita no adoecimento, que fala que você não tem nada" (sic).

Entretanto, essa identidade-doente faz, muitas vezes, com que se vejam presos a um lugar de incapazes ou em discursos que os fazem reféns de uma série de atribuições que a loucura carrega. Se por um lado, a condição de usuários da saúde mental dá acesso a bens simbólicos e benefícios sociais, ela também reforça o estigma 56 .

Na luta pela afirmação de um lugar de legitimidade na sociedade, o reconhecimento de sintomas e diagnósticos, que culminam em tratamentos em que o foco é a doença e não o processo saúde-doença, desconsidera a especificidade da experiência de sofrimento que acomete as diferentes pessoas e torna o sujeito da experiência um sujeito padronizado, sem história, restando apenas a afirmação da necessidade do uso de medicamentos e confirmando uma dinâmica ainda frequente nos CAPS 33.

A partir das discussões nos encontros do grupo intervenção, os usuários perceberam o quão pouco compartilham uns com os outros suas experiências de sofrimento, apesar de terem uma relação de parceria anterior à participação na pesquisa. Essa constatação é índice de que os próprios usuários tomavam o diagnóstico de doentes mentais como o comum entre si. Subsumida em um diagnóstico médico, a experiência de sofrimento dos usuários era homogeneizada e aprisionada em uma identidade-doente. Não à toa, a principal frase do primeiro passo do Guia GAM ("Eu sou uma pessoa, não uma doença!") ecoou sobremaneira nos usuários, reverberando ao longo dos encontros posteriores.

\section{Experiências com a medicação}

As narrativas dos usuários sobre o uso de medicamentos psicotrópicos evidenciam tanto as situações em que a medicação atua como um instrumento a serviço do estar melhor e da ampliação da capacidade de ser e de agir das pessoas e suas práticas, quanto, pelo contrário, as condições em que atua acentuando a experiência de sofrimento.

"É um direito tanto respeitarmos o remédio como também nos respeitarem se quisermos diminuir um psicotrópico, mas desistir não dá certo" (grupo focal narrativo).

Embora a maioria dos usuários estabelecesse uma relação de aparente submissão à prescrição médica, eles faziam uma autogestão do uso à revelia da prescrição médica: controlavam a dosagem, aumentando-a ou diminuindo-a, quando sentiam ser necessário, e suspendiam o uso dos medicamentos em situações em que entendiam que essa suspensão os beneficiaria. Há, portanto, uma gestão do uso, mas experimentada como não podendo ser compartilhada, sobretudo com os profissionais de saúde. A partir das discussões suscitadas nos encontros do grupo intervenção e no grupo focal, constata-se que o espaço destinado à fala dos usuários ainda é insuficiente nos serviços. 
Após o grupo interveção, os usuários perceberam o quão pouco apropriados estavam acerca dos medicamentos de que faziam uso, seus efeitos e o quanto haviam naturalizado a pouca disponibilidade dos profissionais dos CAPS para o acolhimento de suas dúvidas, críticas ou insatisfações a respeito do tratamento medicamentoso. Muitas foram as dificuldades de compreensão com relação às mais variadas informações contidas na bula: a linguagem ali usada parecia pouco ou nada lhes informar acerca do medicamento que usam, carecendo de um trabalho de esclarecimento por parte dos condutores do grupo para a construção de um sentido entre o que ali aparecia informado e suas experiências com os medicamentos. Além disso, em alguns CAPS, não foi fácil conseguirem a bula dos remédios que tomavam.

Quando questionados sobre a participação nos processos decisórios do tratamento, disseram que se acostumaram com uma postura em que o médico é quem sabe sobre medicação. Sentiam que alguns temas não podiam ser falados. Em diferentes momentos do grupo intervenção, os usuários fizeram menção ao descaso que sentiam dos médicos/equipe com relação a experiências com a medicação que tentavam compartilhar, destacando as queixas relacionadas aos efeitos colaterais dos medicamentos. Salientaram que os médicos têm uma "agenda cheia" (sic) e que o curto tempo que têm disponível nos serviços não permite esse tipo de conversa. Questionaram a postura distante de alguns profissionais, que não dialogam, ficando em silêncio nas consultas, sem dar retorno ao que os usuários estão expondo.

No grupo focal narrativa, disseram que os médicos orientam pouco a respeito dos remédios:

“...falam apenas quanto você vai tomar e em que horário. Não conversam sobre como vai ser a adaptação ao remédio" (grupo focal narrativo).

Alguns usuários, mobilizados pelas discussões no grupo interveção, buscaram conversar com seus médicos, visando a ajustes no uso dos medicamentos, ocasiões em que conseguiram fazer algumas adaptações, ou mesmo falar sobre o desejo de não tomar certo tipo de medicamento. Para alguns, a experiência com o Guia GAM não interferiu no uso dos medicamentos, mas mudou seus entendimentos com relação a eles. Os usuários passaram a reconhecer seus saberes com a experiência de uso de medicamentos, e não apenas o saber dos médicos.

Vale destacar uma situação que pode ser considerada como analisadora: em um dos encontros do grupo intervenção, um usuário perguntou aos pesquisadores: "Mas o que vocês querem afinal? Vocês querem que a gente questione os médicos? Que a gente faça perguntas a eles sobre nossa medicação?" O tom da pergunta foi quase de indignação e revelou o que poderia estar se atualizando na relação pesquisadores-usuários como "prescrição": que compartilhassem mais sobre seus medicamentos e sobre o significado deles em suas vidas com os médicos. Externando sua dúvida quanto ao que os condutores do grupo queriam, esse usuário deixou clara a implicação dos condutores com a proposta GAM e o quanto isso estava sendo vivido por ele como uma imposição.

Essa situação aponta a importância do manejo com o grupo ${ }^{54}$, mas também a constante colocação, em análise, da implicação dos pesquisadores, pois a fala desse usuário denunciou uma possível postura prescritiva dos pesquisadores e reivindicou uma relação em que não se repetisse uma experiência de dominação e apropriação de si. Os pesquisadores colocaram, em análise, suas expectativas e o processo que vinha sendo construído no grupo e puderam se reposicionar.

As narrativas indicam que, quando o assunto é medicação, há uma desresponsabilização da equipe, que passa a designar o médico como o único capaz de conversar sobre esse tema 22 . Também apontam o incômodo com a não possibilidade de escolha de quem são seus médicos de referência. Essa decisão é tomada exclusivamente pelas equipes, considerando apenas o critério do território, e não a relação dos usuários com os profissionais do serviço.

\section{Direitos de usuários}

As narrativas dos usuários evidenciam que um dos desafios da reforma psiquiátrica, sobretudo dos CAPS, enquanto articuladores de cuidado, é, justamente, o reconhecimento dos direitos dos usuários. Os usuários perceberam que têm vários direitos, mas, ao mesmo tempo, não os usufruíam. Essa discussão ganhou destacada intensidade quando vinculada ao reconhecimento do direito ao trabalho e ao benefício, indicando, mais uma vez, o quanto a identidade-doente se desdobra em preconceito e gera dificuldades de acesso ao mundo do trabalho. Os usuários expressaram a forte vivência de que 
um direito exclui o outro: se eles têm direito ao benefício pela doença, não conseguem trabalhar; se conseguem trabalhar, não têm direito ao benefício.

"Se é aposentado, não tem direito a emprego. Se vai trabalhar, não consegue. Se fala que faz tratamento, ninguém dá emprego. Que toma remédio (...) é repugnado" (GF0).

A temática dos direitos e da cidadania tem sido apropriada pelos movimentos sociais contemporâneos para formulação de estratégias de empowerment e de reivindicações específicas 57,58. Uma questão que tem sido relevante para o movimento de usuários no campo da saúde mental diz respeito ao fato de que reivindicações por direitos sociais especiais podem conflitar-se e enfraquecer outras lutas no campo dos direitos civis. Em nome dos direitos civis, os usuários lutam contra a segregação e a estigmatização da loucura e pela recuperação de sua capacidade civil para gerir suas vidas. Entretanto, lutam também por direitos sociais especiais, como benefícios pecuniários, esquemas de trabalho especiais, passe livre no transporte público etc. Trata-se de um paradoxo no que diz respeito à coexistência da luta pelos direitos civis e pelos direitos sociais. Uma pesquisa realizada com usuários de saúde mental de uma grande cidade brasileira 59 evidenciou que os usuários só acessaram seus direitos básicos a partir do processo de adoecimento. O paradoxo é justamente a defesa de direitos iguais e, ao mesmo tempo, singulares, fazendo conciliar a universalidade dos direitos civis com a especificidade de certos segmentos da população, como os usuários da saúde mental.

Após a experiência com o Guia GAM, os usuários referiram a necessidade de serem apoiados nos movimentos de reivindicação dos seus direitos, indicando que são movimentos difíceis de serem sustentados sozinhos. A informação acerca dos direitos dos usuários é necessária, mas não suficiente, pois não garante a emergência ou o nascimento de um sujeito de direitos, aquele que tem a experiência de direitos encarnada e que é reconhecido pelo outro como tal. Por isso, não basta que o usuário saiba que pode, por exemplo, recusar a medicação, é também necessário que ele seja legitimado como um sujeito de direitos cuja vontade e decisão devem ser consideradas pelos profissionais de saúde e pela comunidade 9 .

\section{Participação e militância política}

Os usuários não se sentiam participando da gestão de seus tratamentos, nem ouvidos no direito de recusá-los. Ressaltaram a importância dos Conselhos de Saúde como espaços de exercício dessa participação e reivindicação de seus direitos.

Reconheceram a associação da qual cinco dos sete integrantes do grupo intervenção faziam parte como um importante espaço de participação e militância política. Identificaram-na como uma instância de pertencimento e apoio, bem como de função fiscalizadora e de militância contra os manicômios e as práticas manicomiais. Afirmaram que, no processo da pesquisa, aprenderam a se valorizar, pois nele sentiram que o que tinham a dizer era considerado importante e perceberam o quão pouco compartilhavam entre si sobre suas experiências de adoecimento e de uso de medicamentos, apesar da convivência em espaços políticos na cidade.

"E tudo isso que eu aprendi lá [grupo intervenção GAM] eu posso passar pros outros também. Agora eu falo sobre meus tratamentos, sobre meus medicamentos, nos conselhos locais, no grupo que tem dos meus tratamentos lá no CAPS. Falo, por exemplo, que eu não devo terminar os tratamentos por conta própria, que eu tenho direitos e tenho que lutar por eles, não preciso ficar assim, acomodado, esperando tudo pelos outros se eu posso ter um pouquinho de autonomia. Se fez bem pra mim, deve fazer muito bem pros outros pacientes ou para quem quer que esteja tomando remédio e que esteja falando comigo" (narrativa João).

Destacaram o desejo de levar as discussões disparadas pelo Guia para outros usuários da rede por meio de um projeto que construíram durante o processo de pesquisa e que se propunha a constituir grupos para uma discussão sobre saúde mental, utilizando a abordagem GAM. Reconheceram a importância, mas também o desafio, de fazerem valer seus direitos no cotidiano dos seus tratamentos para a efetivação das reivindicações do movimento da Reforma Psiquiátrica nos CAPS.

Cabe salientar, como uma possível limitação do estudo, que essas falas se deram em um contexto em que os usuários estavam participando da pesquisa justamente por terem um diagnóstico de transtorno mental grave, o que pode tê-los influenciado, imaginando uma expectativa dos pesquisadores de que falassem desse lugar. Também não se deve negligenciar o fato de que foi por meio dessa condição de doentes, assim reconhecida e legitimada na sociedade, que eles passaram a ocupar lugares de repre- 
sentação política, como nos conselhos, na associação de que participam e em outros movimentos de que fazem parte. Além disso, trata-se de um perfil de usuários, de modo geral, destoante da maioria dos usuários dos CAPS.

\section{Conclusão}

O uso do Guia GAM no grupo intervenção mostrou-se como um dispositivo com potência de empoderamento dos usuários frente aos seus tratamentos, reforçando sua importância como dispositivo de cogestão e de consequente construção de outras configurações nas relações de poder. Ao mesmo tempo, evidenciou uma postura de ingenuidade e docilidade dos usuários frente aos seus tratamentos, indicando a importância do investimento em espaços de compartilhamentos entre usuários sobre suas experiências de adoecimento e de uso de medicamentos.

Proporcionou reposicionamentos subjetivos tanto nos usuários quanto nos pesquisadores, que constantemente colocavam, em análise, sua implicação com a pesquisa para que não reproduzissem, no grupo, as práticas que o próprio dispositivo se propunha a enfrentar. O desafio de realizar a pesquisa com os usuários fez como que os lugares de usuários e pesquisadores fossem tensionados. Foi preciso que os pesquisadores desconstruíssem a identidade usuário-militante sob o risco de subsumir suas experiências de participação política no campo da saúde mental em uma suposta homogeneidade militante. Nesse sentido, recomendamos fortemente a autocrítica nesse tipo de investigação e o desenho de projetos que incluam dispositivos para que esse tipo de autoanálise possa acontecer.

É importante que outras pesquisas aprofundem a investigação dos efeitos da experimentação do Guia GAM não apenas em usuários, mas também em trabalhadores das equipes de saúde, sobretudo, nos prescritores.

\section{Colaboradores}

L. L. M. Gonçalves realizou a proposta do artigo, a revisão da literatura, a análise dos dados e a redação do artigo. R. T. Onocko Campos orientou a proposta do artigo, a análise dos dados e participou da redação do manuscrito.

\section{Agradecimentos}

À Coordenação de Aperfeiçoamento de Pessoal de Nível Superior (Capes), Brasil, pela bolsa de doutorado concedida a uma das autoras no processo de pesquisa. À Aliança Internacional de Pesquisa Universidade e Comunidade/ARUCI/Brasil, pelo financiamento.

\section{Referências}

1. Yasui S, Costa-Rosa A. A estratégia de atenção psicossocial: desafio na prática dos novos dispositivos em saúde mental. Saúde Debate 2008; 32:27-37.

2. Otanari TMC, Leal EM, Onocko Campos R, Palombini AL, Passos E. Os efeitos na formação de residentes de psiquiatria ao experimentarem grupos GAM. Rev Bras Educ Méd 2011; 35:460-7.

3. Figueiró RA, Dimenstein M. O cotidiano de usuários de CAPS: empoderamento ou captura? Fractal Rev Psicol 2010; 22:431-46.

4. Nunes M, Torrente $M$. Estigma e violências no trato com a loucura: narrativas de centros de atenção psicossocial, Bahia e Sergipe. Rev Saúde Pública 2009; 43:101-8.

5. Miranda L, Oliveira TFK, Santos CTB. Estudo de uma rede de atenção psicossocial: paradoxos e efeitos da precariedade. Psicol Ciênc Prof 2014; 34:592-611. 
6. Bulhões ABC, Vasconcelos MFF, Escócia L. Processos de desinstitucionalização em CAPS-AD como estratégia de humanização da atenção e gestão da saúde. In: Escócia L, Paulon SM, organizadores. Cadernos HumanizaSUS. Volume 5: saúde mental. Brasília: Ministério da Saúde; 2014. p. 41-64.

7. Benevides de Barros R. Reforma psiquiátrica brasileira: resistências e capturas em tempos neoliberais. In: Conselho Federal de Psicologia, organizador. Loucura, ética e política: escritos militantes. São Paulo: Casa do Psicólogo; 2003. p. 196-206.

8. Jacob KS, Sharan P, Mirza J, Garrido-Cumbrera M, Seedat S, Mari JJ, et al. Global mental health 4 - mental health systems in countries: where are we now? Lancet 2007; 370:1061-77.

9. Emerich BF, Onocko Campos R, Passos E. Direitos na loucura: o que dizem usuários e gestores dos Centros de Atenção Psicossocial (CAPS). Interface Comun Saúde Educ (Online) 2014; 18:685-96.

10. Rodriguez del Barrio L, Corin E, Poirel M-L, Drolet M. Limites du rôle de la médication psychiatrique dans le processus de réhabilitation du point de vue des usagers, Pharmacologie et santé mentale. Montréal: Équilibre, Association Canadienne de Santé Mentale; 2006.

11. Amarante P. Saúde mental e atenção psicossocial. Rio de Janeiro: Editora Fiocruz; 2007. (Coleção Temas em Saúde).

12. Faraone S, Barcala A, Torricelli F, Bianchi E, Tamburrino MC. Discurso médico y estrategias de marketing de la industria farmacéutica en los procesos de medicación de la infancia en Argentina. Interface Comun Saúde Educ 2010; 14:485-98

13. Rosa BPGD, Winogard M. Palavras e pílulas: sobre a medicamentalização do mal-estar psíquico na atualidade. Psicol Soc (Impr.) 2011; 23 n. spe:37-44.

14. Bezerra IC, Jorge MSB, Gondim APS, Lima LL, Vasconcelos MGF. "I went to the health unit and the doctor sent me here": process of medicationalization and (non)resolution of mental healthcare within primary care. Interface Comun Saúde Educ (Online) 2014; 18:61-74.

15. Ortega F, Barros D, Caliman L, Itabirahy C, Junqueira L, Ferreira CP. Ritalin in Brazil: production, discourse and practices. Interface Comun Saúde Educ 2010; 14:499-510.

16. Tajima O. Mental health care in Japan: recognition an treatment of depression and anxiety disorders. J Clin Psychiatry 2001; 62 Suppl 13:39-44.

17. Ungvari GS, Chung YG, Chee YK, Fung-Shing N, Kwong TW, Chiu HF. The pharmacological treatment of schizophrenia in chinese patients: a comparison of prescription patterns between 1996 and 1999. Br J Clin Pharmacol 2002; 54:437-44.

18. Cadilhe S. Benzodiazepinas: prevalência de prescrição e concordância com os motivos de consumo. Revista Portuguesa de Medicina Geral e Familiar 2004; 20:193-202.
19. United Nations; International Narcotics Control Board. Psychotropic substances: statistics for 2006: assessments of annual medical and scientific requirement. Geneva: United Nations; 2008.

20. Hull SA, Aquino P. Explaining variation in antidepressant prescribing in east London: a cross sectional study. Farm Pract 2005; 22:37-42.

21. Rodriguez Del Barrio L, Perron N, Ouellette JN. Psicotrópicos e saúde mental: escutar ou regular o sofrimento? In: Onocko Campos R, Furtado JP, Passos E, Benevides R. organozadores. Pesquisa avaliativa em saúde mental: desenho participativo e efeitos de narratividade. São Paulo: Editora Hucitec; 2008. p. 125-51.

22. Onocko Campos R, Palombini AL, Silva AE, Passos E, Leal EM, Serpa Júnior OD, et al. Multicenter adaptation of the guide for autonomous management of medication. Interface Comun Saúde Educ 2012; 16:967-80.

23. Lopes TS, Dahl CM, Serpa JR OD, Leal EM, Campos RTO, Diaz AG. The process of recovery in the perspective of persons with schizophrenia spectrum disorders and of psychiatrists working at psychosocial health care services. Saúde Soc 2012; 21:558-71.

24. Stewart M. Medicina Centrada na Pessoa: transformando o método clínico. Porto Alegre: Editora Artmed; 2010.

25. McWhinney I. Manual de medicina de família e comunidade. Porto Alegre: Editora Artmed; 2010.

26. Greenhalgh T. Narrative based medicine in an evidence based world. BMJ 1999; 318:323-5.

27. Onocko Campos RT, Passos E, Palmobini AL, Santos DVD, Stefanello S, Gonçalves LLM, et al. A Gestão Autônoma da Medicação: uma intervenção analisadora de serviços em saúde mental. Ciênc Saúde Coletiva 2013; 18:2889-98.

28. Regroupement Des Ressources Alternatives En Santé Mentale Du Québec. Balises pour une approche alternative des pratiques de soutien communautaire en santé mentale. Québec: Regroupement des Ressources Alternatives en Santé Mentale du Québec; 2006.

29. Regroupement Des Ressources Alternatives En Santé Mentale Du Québec. Repères pour une Gestion autonome de la médication en santé mentale. Guide d'accompagnement. Montréal: Regroupement des Ressources Alternatives en Santé Mentale du Québec; Équipe de Recherche et d'Action en Santé Mentale et Culture; 2006.

30. Santos DVD. Uso de psicotrópicos na atenção primária no Distrito Sudoeste de Campinas e sua relação com os arranjos da clínica ampliada [Dissertação de Mestrado]. Campinas: Faculdade de Ciências Médicas, Universidade Estadual de Campinas; 2009.

31. Vasconcelos EM. Empoderamento de usuários e familiares em saúde mental em pesquisa avaliativa/interventiva: uma breve comparação entre a tradição anglo-saxônica e a experiência brasileira. Ciênc Saúde Coletiva 2013; 18:2825-35. 
32. Costa DFC, Paulon SM. Participação e protagonismo em saúde mental: a insurgência de um Coletivo. Saúde Debate 2010; 95:572-82.

33. Mielke FB, Kantorski LP, Olschowsky A, Jardim VMR. Características do cuidado em saúde mental em um CAPS na perspectiva dos profissionais. Trab Educ Saúde 2011; 9:265-76.

34. Onocko Campos R, Furtado JP. A participação de diferentes grupos de interesse na geração de conhecimentos sobre a rede de serviços avaliada. In: Onocko Campos R, Furtado JP, Passos E, Benevides R, organizadores. Pesquisa avaliativa em saúde mental: desenho participativo e efeitos de narratividade. São Paulo: Editora Hucitec; 2008. p. 231-48.

35. Alves TC, Oliveira WF, Vasconcelos EM. A visão de usuários, familiares e profissionais acerca do empoderamento em saúde mental. Physis (Rio J.) 2013; 239:51-71.

36. Charles C, Gafni A, Whelan T. Shared decision-making in the medical encounter: what does it mean? (or it takes at least two to tango). Soc Sci Med 1997; 44:681-92.

37. Deegan PE, Drake RE. Shared decision making and medication management in the recovery process. Psychiatr Ser 2006; 57:1636-9.

38. Barry MJ, Edgman-Levitan S. Shared decision making-the pinnacle of patient-centered care. N Engl J Med 2012; 366:780-1.

39. Elwyn G, Frosch D, Thomson R, Joseph-Williams N, Lloyd A, Kinnersley P, et al. Shared decision making: a model for clinical practice. J Gen Intern Med 2012; 27:1361-7.

40. Organización Mundial de la Salud. Advocacy for mental health legislation and human rights. Política de salud mental y paquete de servicios. Geneva: Organización Mundial de la Salud; 2003.

41. World Health Organization. WHO QualityRights tool kit to assess and improve quality and human rights in mental health and social care facilities. Geneva: World Health Organization; 2012.

42. Almeida KS, Dimenstein M, Severo AK. Empoderamento e atenção psicossocial: notas sobre uma associação de saúde mental. Interface Comun Saúde Educ 2010; 14:577-89.

43. Palombini AL, Onocko Campos RT, Silveira M, Gonçalves LLM, Zanchet L, et al. Relations between research and clinical care in co-management studies with mental health care users. Ciênc Saúde Coletiva 2013; 18:2899-908.

44. Passos E, Palombini AL, Onocko Campos R. Estratégia cogestiva na pesquisa e na clínica em saúde mental. ECOS - Estudos Contemporâneos da Subjetividade 2013; 3:4-17.

45. Passos E, Palombini, AL, Onocko Campos R, Rodrigues SE, Melo J, Maggi PM, et al. Autonomia e cogestão na prática em saúde mental: o dispositivo da gestão autônoma da medicação (GAM). Aletheia 2013; (41):24-38.

46. Gatti BA. Grupo focal na pesquisa em ciências sociais e humanas. Brasília: Líber Livro; 2005.
47. Westphal MF, Bógus CM, Faria MM. Grupos focais: experiências precursoras em programas educativos em saúde no Brasil. Bol Oficina Sanit Panam 1996; 120:472-82.

48. Onocko Campos RT, Furtado JP. Narrativas: utilização na pesquisa qualitativa em saúde. Rev Saúde Pública 2008; 42:1090-6.

49. Miranda L, Figueiredo MD, Ferrer AL, Onocko Campos RT. Dos grupos focais aos grupos focais narrativos: uma descoberta no caminho da pesquisa. In: Onocko Campos R, Furtado JP, Passos E, Benevides R, organizadores. Pesquisa avaliativa em saúde mental: desenho participativo e efeitos de narratividade. São Paulo: Aderaldo \& Rothschild; 2008. p. 249-77.

50. Onocko Campos R. Fale com eles! O trabalho interpretativo e a produção de consenso na pesquisa qualitativa em saúde: inovações a partir de desenhos participativos. Physis (Rio J.) 2011;21:1269-86.

51. Minayo MCS, Deslandes SF, Gomes R. Pesquisa social: teoria, método e criatividade. Petrópolis: Editora Vozes; 2007.

52. Ricoeur P. Interpretação e ideologias. Rio de Janeiro: Editora F. Alves; 1990.

53. Ricoeur P. Tempo e narrativa. Campinas: Papirus Editora; 1997.

54. Ricoeur P. A vida: uma narrativa em busca de narrador. Escritos e conferências I: em torno da psicanálise. São Paulo: Editora Loyola; 2010.

55. Onocko Campos RT. O exercício interpretativ. In: In: Onocko Campos R, Furtado JP, Passos E, Benevides R, organizadores. Pesquisa avaliativa em saúde mental: desenho participativo e efeitos de narratividade. São Paulo: Aderaldo \& Rothschild; 2008. p. 278-99.

56. Goffman E. Estigma: notas sobre a manipulação da identidade deteriorada. São Paulo: LTC Editora; 1988

57. Vasconcelos EM. O poder que brota da dor e da opressão: empowerment, sua história, teorias e estratégias. São Paulo: Editora Paulus; 2003.

58. Vasconcelos EM. Reinvenção da cidadania no campo da saúde mental e estratégia política no movimento de usuários. In: Santos LC, Pereira ICG, Bisneto JA, Vasconcelos EM, organizadores. Saúde mental e serviço social - o desafio da subjetividade e da interdisciplinaridade. São Paulo: Cortez Editora; 2008. p. 69-95.

59. Surjus LTLS, Onocko Campos R. A avaliação dos usuários sobre os Centros de Atenção Psicossocial (CAPS) de Campinas, SP/Brasil. Rev Latinoam Psicopatol Fundam 2011; 14:122-33. 


\section{Abstract}

Numerous studies have highlighted the tendency to chronicity of treatments centered on the use of medications. This study was conducted in a large Brazilian city with users of Centers for Psychosocial Care (CAPS), with the aim of evaluating the effects of experimenting with the personal Guide for Autonomous Management of Medication (GAM) and the users' relationship to their treatments and participation. The instrument, created in Canada and translated and adapted to Brazil, was tested in intervention groups with users of CAPS with severe mental disorders and a history of political participation in the field of health. Focus groups and in-depth interviews were performed. The transcriptions were transformed into narratives, and four analytical categories were defined: subjects with radical experiences of suffering; experiences with medication; users' rights; and participation and political activism. In testing GAM, users expressed a tension between reproduction of illness-centered identity and the legitimate uniqueness of their personal experiences. They showed greater knowledge of the medications they were taking, began to recognize their own expertise in their use of medications, and some sought adjustments to their treatment. They reported the need for support in claiming their rights and strengthening their participation as mental health activists. In conclusion, the Brazilian version of the GAM guide has the potential to contribute to users' empowerment, thus allowing dialogue between the results in mental health care in Brazil and the international scenario.

Mental Health Services; Drug Utilization; Citizen Participation; Personal Autonomy

\section{Resumen}

Numerosos estudios apuntan la tendencia a la cronicidad de los tratamientos cuando se encuentran centrados en el uso de medicamentos. Esta investigación se realizó en una gran ciudad brasileña, con pacientes de Centros de Atención Psicosocial (CAPS), con el objetivo de evaluar los efectos de la experimentación de la Guía personal de la Gestión Autónoma de la Medicación (Guía GAM) en la relación de estos usuarios con sus tratamientos y en sus acciones de participación. Este instrumento, creado en Canadá, fue traducido y adaptado para Brasil, fue experimentado, mediante grupos de intervención, con pacientes de CAPS con trastorno mental grave y con un historial de participación política en el campo de la salud. Se realizaron grupos focales y entrevistas en profundidad. Las transcripciones se transformaron en narraciones, y fueron definidas cuatro categorías de análisis: sujetas a experiencias radicales de sufrimiento; experiencias con la medicación; derechos de pacientes; participación y militancia política. Con la experimentación de la Guía GAM, los usuarios expresaron una tensión entre la reproducción de la identidad, centrada en la enfermedad y la legitimidad de la singularidad de sus experiencias personales. Demostraron un mayor conocimiento sobre los medicamentos que tomaban, pasaron a reconocer su propia pericia con el uso de medicamentos, $y$ algunos buscaron ajustes en el tratamiento. Informaron de la necesidad de ser apoyados por los movimientos de reivindicación de sus derechos y fortalecieron sus participaciones como militantes de la salud mental. Se concluye que la versión brasileña de la Guía GAM tiene potencial de contribuir con el empoderamiento de los usuarios, permitiendo así una interlocución de las evaluaciones de resultados en salud mental con el escenario internacional.

Servicios de Salud Mental; Utilización de Medicamentos; Participación Ciudadana; Autonomía Personal
Recebido em 26/Set/2016

Versão final reapresentada em 24/Nov/2016

Aprovado em 01/Fev/2017 